\title{
DIVERSIDADE GENÉTICA ENTRE ACESSOS DE SOJA TIPO ALIMENTO COM BASE NO ALGORITMO DE GOWER
}

Luiz Junior Perini ${ }^{1}$, Douglas Mariani Zeffa ${ }^{2}$, Gustavo Henrique Freiria ${ }^{1}$, Paulo Sérgio Novais ${ }^{3}$, Cassio Egidio Cavenaghi Prete ${ }^{1}$

${ }^{1}$ Universidade Estadual de Londrina - UEL, Londrina, Paraná, Brasil. ${ }^{2}$ Universidade Estadual de Maringá - UEM, Maringá, Paraná, Brasil. ${ }^{3}$ GDM Seeds, Cambé, Paraná, Brasil.

\section{RESUMO}

A avaliação simultânea de características qualitativas e quantitativas é uma excelente ferramenta na estimação da divergência genética entre acessos de bancos de germoplasma. O presente estudo objetivou caracterizar e estimar a divergência genética entre acessos de soja tipo alimento com base em descritores qualitativos e quantitativos por meio do algoritmo de Gower, além de identificar possíveis combinações promissoras entre os acessos avaliados. Foram estudados 64 genótipos de soja, sendo 60 acessos de soja tipo alimento, dois padrões comerciais também destinados à alimentação humana e dois padrões comerciais tipo grão. Três características qualitativas e sete quantitativas foram utilizadas para estimar a divergência genética entre os genótipos. 0 experimento foi conduzido na área experimental da Universidade Estadual de Londrina durante a safra 2015/2016, utilizando o delineamento de blocos completos ao acaso, com quatro repetições. Foi observada a presença de variabilidade genética para todas as características avaliadas. A característica produtividade de grãos foi a mais eficiente na discriminação dos acessos avaliados, enquanto que a característica altura de planta apresentou menor contribuição. O método UPGMA apresentou o maior coeficiente de correlação cofenética, permitindo a formação de seis diferentes grupos. As hibridações AL06 × AL22 e AL07 × AL22 são promissoras para obtenção de genótipos superiores destinados ao consumo in natura e produção de leite de soja e tofu. A combinação AL13 × AL60 é considerada favorável para obtenção de genótipos destinados à produção de natto. A utilização dos acessos AL12 e AL13 como genitores é vantajosa para obtenção de genótipos destinados à produção de kuroname.

Palavras-chave: Glycine max L.; análise multivariada; descritores qualitativos; descritores quantitativos.

\section{GENETIC DIVERSITY AMONG FOOD TYPE SOYBEAN ACCESSIONS BASED ON GOWER ALGORITHM}

\section{ABSTRACT}

The simultaneous evaluation of qualitative and quantitative traits is an excellent tool to estimate the genetic divergence among accessions of germplasm banks. The present study aimed to characterize and estimate the genetic divergence among soybean food type accessions based on Gower algorithm and identifies promising combination among accessions evaluated. A total of 64 soybean genotypes were studied, which 60 are food type soybean accessions, two are commercial cultivars also for human consumption, and two are grain type commercial cultivars. Three qualitative and seven quantitative traits were used to estimate the genetic divergence among genotypes. The experiment was carried out in the experimental area of the Universidade Estadual de Londrina during 2015/2016 season, using a randomized complete block design with four repetitions. It was observed the presence of genetic variability to all traits evaluated. The grain yield trait was the most efficient in discriminating the evaluated accessions, while plant height showed lower contribution. The UPGMA method showed the highest coefficient of cophenetic correlation allowing the formation of six different groups. The crossings AL06 $\times$ AL22 and AL07 $\times$ AL22 are promising for obtaining superior genotypes destined to the in natura consumption and production of soy milk and tofu. The combination AL13 $\times$ AL60 is considered favorable for obtaining genotypes destined to natto production. The use of the AL12 and AL13 accessions as parents is advantageous for obtaining genotypes for the production of kuroname.

Keywords: Glycine max L.; multivariate analysis; qualitative descriptors; quantitative descriptors. 


\section{INTRODUÇÃO}

A soja [Glycine max (L.) Merril] é considerado um alimento nutritivo que, além das suas funções nutricionais, pode desempenhar importante papel funcional no organismo com ação benéfica à saúde pela prevenção do aparecimento de diversas doenças (HEA; CHEN, 2017). Embora menos de 5\% da produção mundial de soja sejam destinados ao consumo humano, nichos de mercado para soja tipo alimento vêm crescendo no mundo todo, principalmente pelas suas inúmeras formas de utilização como, por exemplo, na forma de brotos, tofu (queijo de soja), natto (fermentada), edamame (vagens imaturas), kuromame (soja doce) e saladas (YOKOMIZO et al., 2000; HIRAKURI; LAZAROTTO, 2014; CARRÃO-PANIZZI et al., 2016).

A caracterização e avaliação de bancos de germoplasma são realizadas tanto para características qualitativas quanto para características quantitativas, porém as análises e interpretações de forma independente entre esses dois tipos de variáveis podem resultar em uma incompleta distinção entre os acessos avaliados. Dessa forma, a análise conjunta dessas características pode fornecer melhor indicação da potencialidade da variabilidade genética existente nos bancos de germoplasma (MOURA et al., 2010). Por outro lado, são poucos os trabalhos que têm utilizado tal estratégia, seja pela falta de conhecimento de técnicas estatísticas que permitem a utilização dessa abordagem ou pela tendência dos pesquisadores em dar maior importância àquelas variáveis diretamente relacionadas com caracteres trabalhados em programas de melhoramento (GONÇALVES et al., 2008). Além disso, as características qualitativas, principalmente as relacionadas com vagens $e$ grãos, apresentam maior importância em programas de melhoramento de soja tipo alimento em relação à soja tipo grão por estarem relacionadas à aceitabilidade do consumidor e indústria de processamento (SEDIYAMA, 2009).

Gower (1971) propôs uma técnica que permite a análise de dados quantitativos e qualitativos de forma simultânea. Tal método permite que os valores da matriz de distância fiquem compreendidos entre 0 e 1 , sendo necessária a padronização das variáveis quantitativas e qualitativas. Trabalhos utilizando tal abordagem já foram relatados para tomate (Solanum lycopersicum) (ROCHA et al., 2010), quiabo (Abelmoschus esculentus) (KYRIAKOPOULOU et al., 2014), abóbora (Cucurbita moschata) (OLIVEIRA et al., 2016) e soja tipo grão (Glycine max L.) (SILVA et al., 2016). Nesse sentido, o presente trabalho objetivou caracterizar e estimar a divergência genética entre acessos de soja alimento com base em descritores qualitativos e quantitativos, bem como identificar combinações promissoras entre os acessos avaliados.

\section{MATERIAIS E MÉTODOS}

O experimento foi conduzido na área experimental da Universidade Estadual de Londrina (UEL), Londrina, Paraná, Brasil (2319'42"S, $51^{\circ} 12^{\prime} 11^{\prime \prime} \mathrm{W}$ ) durante o ano agrícola de 2015/2016. Foram avaliados 64 genótipos pertencentes ao Banco de Germoplasma de Soja da UEL, sendo 60 acessos de soja tipo alimento, dois cultivares comerciais tipo alimento (BRS 257 e BRS 258) e dois cultivares tipo grão (NK 7059 e BMX Potência). Utilizou-se o delineamento experimental de blocos ao acaso com três repetições. As parcelas foram constituídas de quatro linhas de $4 \mathrm{~m}$ de comprimento com espaçamento de $0,5 \mathrm{~m}$ entre si, sendo consideradas como área útil as duas linhas centrais, desprezando-se $0,5 \mathrm{~m}$ de cada extremidade.

Foram avaliadas as seguintes características: i) cor do tegumento; ii) cor do hilo; iii) hábito de crescimento; iv) altura de planta (ALT, $\mathrm{cm}$ ); v) altura de inserção da primeira vagem (APV, $\mathrm{cm}$ ); vi) produção de grãos por parcela (PRO, g parcela $^{-1}$ ); vii) peso de 100 sementes (PCS, g); viii) número de dias para maturidade (NDM); ix) índice de acamamento (ACA), caráter avaliado no estádio da planta por meio de uma escala de notas visuais, variando de 1 (todas as plantas eretas) a 5 (todas as plantas acamadas); e $\mathrm{x}$ ) valor agronômico (VAG), avaliado no estádio R8 de desenvolvimento da planta, por meio de uma escala de notas visuais, variando de 1 (plantas com características agronômicas ruins) a 5 (plantas com ótimas características agronômicas). Os estádios fenológicos mencionados seguiram à escala proposta por Fehr e Caviness (1977).

As características quantitativas foram submetidas à análise de variância univariada (ANOVA). Quando constatado efeito significativo de tratamento, as médias foram agrupadas pelo 
teste de Scott e Knott (1974) ao nível de 5\% de probabilidade. A importância relativa das características estudadas foi realizada pelo método proposto por Singh (1981). A determinação da distância genética entre as características qualitativas e quantitativas foi realizada por meio do algoritmo de Gower (1971), expresso da seguinte maneira:

$$
S i j=\sum_{k=1}^{p} W i j k . S i j k / \sum_{k=1}^{p} W i j k
$$

em que: $\mathrm{K}$ é o número de variáveis $(\mathrm{k}=1,2, \ldots ; \mathrm{p}=$ número total de características avaliadas); i e j, dois indivíduos quaisquer; $W_{i \mathrm{ijk}}$ é um peso dada a comparação ijk, atribuindo valor 1 para comparações válidas e valor 0 para comparações inválidas (quando o valor da variável está ausente em um ou ambos indivíduos); $S_{\mathrm{ijk}}$ é a contribuição da variável k na similaridade entre os indivíduos i e $\mathrm{j}$, possuindo valores entre 0 e 1 . Para uma variável nominal, se o valor da variável k é a mesma para ambos os indivíduos, i e j, então $S_{\mathrm{ijk}}=1$, caso contrário, é igual a 0 ; para uma variável contínua $S_{i j k}=1-\left|x_{i k}-x_{j k}\right| / R_{k}$ em que $x_{i k}$ e $x_{j k}$ são os valores da variável $k$ para os indivíduos $i \mathrm{e} j$, respectivamente, e $R_{k}$ é a amplitude de variação da variável $k$ na amostra. A divisão por $R_{k}$ tem como função a eliminação das diferenças entre as escalas das variáveis, produzindo um valor dentro do intervalo $[0,1]$ e pesos iguais.

Os agrupamentos hierárquicos dos acessos foram obtidos pelos métodos UPGMA, Ward e Vizinho Mais Próximo. O método de Mojena (1977), que é baseado no tamanho relativo dos níveis de fusão do dendrograma, foi utilizado para indicação do ponto de corte no dendrograma, considerando $k=1,25$ como regra de parada na definição do número de grupos, conforme sugerido por Milligan e Cooper (1985). A validação dos agrupamentos foi determinada pelo coeficiente de correlação cofenética (SOKAL; ROHLF, 1962) e sua significância calculada pelos testes t e de Mantel (1967).

As análises de variância (ANOVA), o teste de agrupamento de médias e o cálculo da importância relativa das características foram realizados com o auxílio do programa Genes (CRUZ, 2016). O programa $R$ (https://www.r-project.org) foi utilizado para as análises de distância genética, agrupamentos hierárquicos, ponto de corte no dendrograma e correlação cofenética.

\section{RESULTADOS E DISCUSSÃO}

Foi observado polimorfismo para todas as características qualitativas avaliadas (Tabela 1). A característica cor de hilo foi a que apresentou maior variação, sendo as cores marrom claro (34,4\% dos acessos), amarela (32,8\%) e marrom $(29,7 \%)$ as mais predominantes. Todavia, a coloração preto imperfeito $(3,1 \%)$ também foi observada. Em geral, hilos de coloração clara (amarela e marrom claro) são preferidos pela indústria de processamento, uma vez que não geram alterações de coloração em seus subprodutos (SEDIYAMA, 2009). Para hábito de crescimento, houve predominância dos acessos do tipo determinado $(70,3 \%)$ em relação aos indeterminados $(28,1 \%)$, sendo que apenas um acesso (AL23) apresentou tipo de crescimento semi-determinado. Para a característica cor do tegumento, a maioria dos acessos $(96,8 \%)$ apresentou cor amarela, enquanto nos demais $(3,2 \%)$ foram observadas coloração marrom. 
Tabela 1. Origem, características qualitativas e médias aritméticas de 64 genótipos de soja para sete variáveis quantitativas agrupadas pelo teste de Scott e Knott em nível de $5 \%$ de probabilidade.

\begin{tabular}{|c|c|c|c|c|c|c|c|c|c|c|c|}
\hline Acesso & Origem & CT & $\mathrm{CH}$ & $\mathrm{HC}$ & ALT & APV & PRO & PCS & NDM & ACA & VAG \\
\hline AL01 & $\mathrm{FP} \times \mathrm{BRS} 216$ & $A$ & $\mathrm{MC}$ & $\mathrm{D}$ & $110,17 \mathrm{c}$ & $16,58 \mathrm{a}$ & $423,6 \mathrm{c}$ & $12,80 \mathrm{i}$ & $133 c$ & $4,00 a$ & $2,13 d$ \\
\hline \multirow[t]{2}{*}{ AL02 } & ARA $\times$ BRS 155 & A & $M$ & 1 & $89,50 \mathrm{~d}$ & $7,00 \mathrm{~b}$ & $825,7 a$ & $17,39 \mathrm{e}$ & $125 \mathrm{~g}$ & $1,78 \mathrm{~d}$ & $3,03 \mathrm{~b}$ \\
\hline & BRS $216 x$ & $A$ & MC & & & & & & & & \\
\hline \multirow[t]{2}{*}{ AL03 } & Wilami & & & D & $94,25 d$ & $13,75 \mathrm{a}$ & $299,4 \mathrm{c}$ & $11,11 \mathrm{j}$ & $121 \mathrm{i}$ & $4,30 \mathrm{a}$ & $2,18 d$ \\
\hline & BRS $216 \times$ & $A$ & MC & & & & & & & & \\
\hline AL04 & Wilami & & & D & $88,50 \mathrm{~d}$ & $14,25 \mathrm{a}$ & $346,5 \mathrm{c}$ & $11,55 \mathrm{j}$ & $123 \mathrm{~h}$ & $3,63 \mathrm{~b}$ & $2,35 d$ \\
\hline AL05 & GA115 × LND31 & $A$ & $M$ & D & $98,92 \mathrm{c}$ & $12,75 \mathrm{a}$ & $575,3 \mathrm{~b}$ & $17,15 \mathrm{e}$ & $121 \mathrm{i}$ & $2,75 \mathrm{c}$ & $2,65 \mathrm{c}$ \\
\hline AL06 & $54 \times 72$ F9:2 & A & A & D & $76,42 \mathrm{f}$ & $11,00 \mathrm{~b}$ & 797,9 a & $23,56 \mathrm{~b}$ & $123 \mathrm{~h}$ & $2,25 \mathrm{~d}$ & $2,85 \mathrm{~b}$ \\
\hline AL07 & $54 \times 72$ F9:2 & $A$ & A & D & $76,75 f$ & $8,67 \mathrm{~b}$ & $811,2 \mathrm{a}$ & $23,98 \mathrm{~b}$ & $123 \mathrm{~h}$ & $1,78 d$ & $3,03 b$ \\
\hline AL08 & Wilami $\times$ FP & $A$ & $M$ & D & $95,08 d$ & $8,67 b$ & $643,9 a$ & 26,83 a & $129 \mathrm{e}$ & $2,50 \mathrm{c}$ & $2,55 \mathrm{c}$ \\
\hline \multirow[t]{2}{*}{ AL09 } & Wilami $\times F P$ & A & $\mathrm{M}$ & D & $76,92 \mathrm{f}$ & $9,25 \mathrm{~b}$ & $326,9 \mathrm{c}$ & $22,30 \mathrm{c}$ & $129 \mathrm{e}$ & $2,65 c$ & $2,20 \mathrm{~d}$ \\
\hline & Toffu I × BRS & A & A & & & & & & & & \\
\hline \multirow[t]{2}{*}{ AL10 } & 216 & & & 1 & $74,25 f$ & $7,25 b$ & $579,8 \mathrm{~b}$ & $17,89 \mathrm{e}$ & $121 \mathrm{i}$ & $1,88 \mathrm{~d}$ & $2,83 \mathrm{~b}$ \\
\hline & $A P \times B R M$ & A & A & D & & & & & & & \\
\hline AL11 & 42660 & & & & $88,92 \mathrm{~d}$ & $8,92 \mathrm{~b}$ & $539,1 b$ & $18,56 \mathrm{e}$ & $121 \mathrm{i}$ & $2,75 \mathrm{c}$ & $2,68 \mathrm{c}$ \\
\hline AL12 & Wilami $\times$ AP & $M$ & $M$ & D & $89,42 d$ & $11,25 b$ & 647,3 a & $19,87 d$ & $121 \mathrm{i}$ & $2,33 \mathrm{c}$ & $2,98 b$ \\
\hline AL13 & Wilami $\times$ AP & $M$ & MC & D & $94,92 \mathrm{~d}$ & $15,25 \mathrm{a}$ & $732,7 \mathrm{a}$ & $11,90 \mathrm{j}$ & $133 c$ & $2,35 \mathrm{c}$ & $3,35 \mathrm{a}$ \\
\hline AL14 & $21 \times 9$ RC2F10 & A & $\mathrm{PI}$ & D & 180,67 a & $7,00 \mathrm{~b}$ & $580,6 b$ & $11,08 \mathrm{j}$ & $125 \mathrm{~g}$ & $3,58 \mathrm{~b}$ & $2,10 \mathrm{~d}$ \\
\hline AL15 & $21 \times 9$ RC2F10 & $A$ & $\mathrm{PI}$ & D & 83,17 e & $9,67 b$ & $553,3 \mathrm{~b}$ & $11,53 \mathrm{j}$ & $125 \mathrm{~g}$ & $2,45 c$ & $2,45 \mathrm{c}$ \\
\hline AL16 & $21 \times 9$ RC2F10 & $A$ & $M$ & I & 169,83 a & $5,08 \mathrm{~b}$ & $389,5 \mathrm{c}$ & 8,251 & $130 d$ & $4,18 \mathrm{a}$ & $1,95 d$ \\
\hline AL17 & $72 \times 55$ & A & $\mathrm{MC}$ & D & $94,75 d$ & $11,75 \mathrm{a}$ & $558,3 \mathrm{~b}$ & $13,04 i$ & $125 \mathrm{~g}$ & $2,75 \mathrm{c}$ & $2,55 \mathrm{c}$ \\
\hline AL18 & BR $92 \times F P$ & $A$ & $M$ & D & $115,00 \mathrm{~b}$ & $14,84 \mathrm{a}$ & $563,2 b$ & $13,17 i$ & $136 b$ & $1,83 d$ & $3,08 b$ \\
\hline AL19 & ARA $\times$ BRS 155 & $A$ & $M$ & D & $96,50 d$ & $14,33 \mathrm{a}$ & $631,2 \mathrm{a}$ & $15,29 \mathrm{~g}$ & $123 \mathrm{~h}$ & $2,73 \mathrm{c}$ & $2,65 \mathrm{c}$ \\
\hline AL20 & ARA $\times$ BRS 155 & $A$ & $M$ & I & 105,09 c & $12,92 \mathrm{a}$ & $582,0 \mathrm{~b}$ & $13,76 \mathrm{i}$ & $123 \mathrm{~h}$ & $2,85 \mathrm{c}$ & $2,55 \mathrm{c}$ \\
\hline AL21 & ARA $\times$ BRS 155 & $A$ & A & D & $92,09 d$ & $14,08 \mathrm{a}$ & $834,4 a$ & $15,58 \mathrm{~g}$ & $121 \mathrm{i}$ & $2,50 \mathrm{c}$ & $3,13 b$ \\
\hline AL22 & Wilami $\times$ FP & $A$ & A & D & $90,92 d$ & $18,67 \mathrm{a}$ & $372,0 \mathrm{c}$ & $21,10 \mathrm{c}$ & $123 \mathrm{~h}$ & $3,88 \mathrm{a}$ & $2,18 d$ \\
\hline \multirow[t]{2}{*}{ AL23 } & Toffu I × ARA & $A$ & A & SD & $120,00 \mathrm{~b}$ & $13,00 \mathrm{a}$ & $627,7 \mathrm{a}$ & $16,56 f$ & $129 \mathrm{e}$ & $2,98 \mathrm{c}$ & $2,55 \mathrm{c}$ \\
\hline & BRS $155 \times$ BRS & $A$ & A & & & & & & & & \\
\hline \multirow[t]{2}{*}{ AL24 } & 213 & & & 1 & $94,25 d$ & $11,59 \mathrm{a}$ & 685,9 a & $17,81 \mathrm{e}$ & $121 \mathrm{i}$ & $2,13 d$ & $2,85 \mathrm{~b}$ \\
\hline & BRS $155 \times$ BRS & A & A & D & & & & & & & \\
\hline \multirow[t]{2}{*}{ AL25 } & 213 & & & & $94,83 \mathrm{~d}$ & $15,09 \mathrm{a}$ & $526,8 \mathrm{~b}$ & $13,91 \mathrm{i}$ & $121 \mathrm{i}$ & $2,08 \mathrm{~d}$ & $3,33 \mathrm{a}$ \\
\hline & BRS $155 \times$ BRS & $A$ & A & D & & & & & & & \\
\hline \multirow[t]{2}{*}{ AL26 } & 216 & & & & $90,42 \mathrm{~d}$ & $11,84 \mathrm{a}$ & $690,2 \mathrm{a}$ & $15,68 \mathrm{~g}$ & $121 \mathrm{i}$ & $2,05 d$ & $3,00 \mathrm{~b}$ \\
\hline & BRS $155 \times$ BRS & $A$ & A & & & & & & & & \\
\hline \multirow[t]{2}{*}{ AL27 } & 216 & & & I & $91,50 \mathrm{~d}$ & $18,50 \mathrm{a}$ & $416,0 \mathrm{c}$ & $13,10 i$ & $128 \mathrm{f}$ & $3,13 b$ & $2,33 d$ \\
\hline & BRS $155 \times$ BRS & $A$ & MC & D & & & & & & & \\
\hline AL28 & 216 & & & & $77,34 \mathrm{f}$ & $11,42 b$ & $604,4 b$ & $15,14 \mathrm{~h}$ & $125 \mathrm{~g}$ & $2,45 c$ & $2,73 \mathrm{c}$ \\
\hline AL29 & BRS $213 \times \mathrm{FP}$ & $A$ & MC & D & $91,33 \mathrm{~d}$ & $14,00 \mathrm{a}$ & $711,3 \mathrm{a}$ & $10,82 \mathrm{j}$ & $128 \mathrm{f}$ & $2,33 \mathrm{c}$ & $2,60 \mathrm{c}$ \\
\hline AL30 & BRS $213 \times F P$ & A & A & D & $85,42 d$ & $18,08 \mathrm{a}$ & $564,4 \mathrm{~b}$ & $11,70 \mathrm{j}$ & $125 \mathrm{~g}$ & $2,00 \mathrm{~d}$ & $2,80 \mathrm{~b}$ \\
\hline \multirow[t]{2}{*}{ AL31 } & BRS $213 \times$ ARA & $A$ & A & D & $73,17 f$ & $14,58 \mathrm{a}$ & 679,3 a & $15,65 \mathrm{~g}$ & $125 \mathrm{~g}$ & $1,83 d$ & $2,90 \mathrm{~b}$ \\
\hline & BRS $213 \times$ BRS & A & A & D & & & & & & & \\
\hline \multirow[t]{2}{*}{ AL32 } & 155 & & & & $86,67 d$ & $12,58 \mathrm{a}$ & $525,0 \mathrm{~b}$ & $13,68 i$ & $125 \mathrm{~g}$ & $2,70 \mathrm{c}$ & $2,80 \mathrm{~b}$ \\
\hline & BRS $213 \times$ BRS & A & & D & & & & & & & \\
\hline AL33 & 155 & & MC & & $84,42 \mathrm{e}$ & $13,67 \mathrm{a}$ & $563,4 \mathrm{~b}$ & $14,97 \mathrm{~h}$ & $123 \mathrm{~h}$ & $2,18 d$ & $2,75 \mathrm{c}$ \\
\hline AL34 & BRS $216 \times F P$ & $A$ & $M$ & D & $85,34 \mathrm{~d}$ & $9,58 \mathrm{~b}$ & $661,4 a$ & $16,48 \mathrm{f}$ & $138 a$ & $2,08 d$ & $2,63 \mathrm{c}$ \\
\hline AL35 & BRS $216 \times F P$ & A & $\mathrm{MC}$ & D & $85,84 \mathrm{~d}$ & $15,00 \mathrm{a}$ & $361,9 \mathrm{c}$ & $16,81 \mathrm{f}$ & $125 \mathrm{~g}$ & $3,38 \mathrm{~b}$ & $2,33 d$ \\
\hline \multirow[t]{2}{*}{ AL36 } & BRS $216 \times F P$ & $A$ & A & D & $88,92 \mathrm{~d}$ & $13,75 \mathrm{a}$ & $361,7 \mathrm{c}$ & $17,11 \mathrm{e}$ & $128 \mathrm{f}$ & $4,00 \mathrm{a}$ & $2,18 d$ \\
\hline & BRS $216 \times$ & $A$ & A & D & & & & & & & \\
\hline AL37 & Wilami & & & & $83,84 \mathrm{e}$ & $10,50 \mathrm{~b}$ & $661,4 a$ & $14,01 \mathrm{i}$ & $121 \mathrm{i}$ & $2,03 d$ & $2,95 \mathrm{~b}$ \\
\hline AL38 & $\mathrm{BRM} 92 \times \mathrm{AP}$ & A & A & D & $86,17 d$ & $11,67 \mathrm{a}$ & $523,2 \mathrm{~b}$ & $15,68 \mathrm{~g}$ & $130 \mathrm{~d}$ & $2,83 c$ & $2,73 c$ \\
\hline
\end{tabular}




\begin{tabular}{|c|c|c|c|c|c|c|c|c|c|c|c|}
\hline AL39 & BRM $92 \times$ AP & A & $A$ & $\mathrm{D}$ & $83,33 \mathrm{e}$ & $7,92 b$ & $624,7 \mathrm{a}$ & $15,32 \mathrm{~g}$ & $125 \mathrm{~g}$ & $2,20 d$ & $2,85 b$ \\
\hline$A L 40$ & BRM $92 \times A P$ & $A$ & $\mathrm{MC}$ & $\mathrm{D}$ & $92,58 d$ & $12,00 \mathrm{a}$ & $550,7 \mathrm{~b}$ & $14,98 \mathrm{~h}$ & $129 \mathrm{e}$ & $3,38 b$ & $2,50 \mathrm{c}$ \\
\hline AL41 & BRM $92 \times$ AP & $A$ & $A$ & $\mathrm{D}$ & $85,50 \mathrm{~d}$ & $8,67 \mathrm{~b}$ & $665,4 \mathrm{a}$ & $14,66 \mathrm{~h}$ & $121 \mathrm{i}$ & $2,48 c$ & $2,63 \mathrm{c}$ \\
\hline AL42 & BRM $92 \times$ AP & $A$ & $\mathrm{MC}$ & $\mathrm{D}$ & $99,34 \mathrm{c}$ & $10,83 \mathrm{~b}$ & $485,1 \mathrm{~b}$ & $11,69 \mathrm{j}$ & $129 \mathrm{e}$ & $4,00 \mathrm{a}$ & $2,38 d$ \\
\hline \multirow[t]{2}{*}{ AL43 } & BRM $92 \times A P$ & $A$ & $M$ & 1 & $92,92 \mathrm{~d}$ & $9,67 b$ & 699,6 a & $16,84 \mathrm{f}$ & $123 \mathrm{~h}$ & $2,20 d$ & $2,75 \mathrm{c}$ \\
\hline & $\mathrm{BR} 36 \times \mathrm{BRS}$ & $A$ & & 1 & & & & & & & \\
\hline AL44 & 258 & & $\mathrm{MC}$ & & $82,17 \mathrm{e}$ & $9,84 \mathrm{~b}$ & 749,0 a & $18,14 \mathrm{e}$ & $125 \mathrm{~g}$ & $1,98 d$ & $3,00 \mathrm{~b}$ \\
\hline \multirow[t]{2}{*}{ AL45 } & BR $36 \times$ MN01 & $A$ & $A$ & 1 & $102,08 \mathrm{c}$ & $10,67 \mathrm{~b}$ & $560,3 \mathrm{~b}$ & $17,63 \mathrm{e}$ & $121 \mathrm{i}$ & $2,28 d$ & $2,60 \mathrm{c}$ \\
\hline & BRS $213 \times$ BR & $A$ & $\mathrm{MC}$ & 1 & & & & & & & \\
\hline \multirow[t]{2}{*}{ AL46 } & 36 & & & & $71,83 \mathrm{f}$ & $8,58 \mathrm{~b}$ & 722,9 a & $19,74 \mathrm{~d}$ & $125 \mathrm{~g}$ & $1,38 d$ & $2,63 c$ \\
\hline & BRS $216 \times$ BRS & $A$ & $\mathrm{MC}$ & $\mathrm{D}$ & & & & & & & \\
\hline \multirow[t]{2}{*}{ AL47 } & 213 & & & & $91,67 d$ & $11,33 \mathrm{~b}$ & $526,9 \mathrm{~b}$ & $12,89 \mathrm{i}$ & $121 \mathrm{i}$ & $2,80 \mathrm{c}$ & $2,45 \mathrm{c}$ \\
\hline & BRS $216 \times$ BRS & $A$ & $\mathrm{MC}$ & $\mathrm{D}$ & & & & & & & \\
\hline \multirow[t]{2}{*}{ AL48 } & 258 & & & & $86,42 \mathrm{~d}$ & $8,75 \mathrm{~b}$ & 619,4 a & 10,88 j & $128 \mathrm{f}$ & $2,70 \mathrm{c}$ & $2,48 \mathrm{c}$ \\
\hline & BRS $216 \times$ BRS & $A$ & $\mathrm{MC}$ & 1 & & & & & & & \\
\hline \multirow{2}{*}{ AL49 } & 258 & & & & $86,25 d$ & $13,83 \mathrm{a}$ & $573,3 \mathrm{~b}$ & $10,07 \mathrm{k}$ & $125 \mathrm{~g}$ & $2,03 d$ & $2,75 \mathrm{c}$ \\
\hline & BRS $216 \times$ BRS & $A$ & $\mathrm{MC}$ & 1 & & & & & & & \\
\hline \multirow[t]{2}{*}{ AL50 } & 258 & & & & $82,25 \mathrm{e}$ & $12,50 \mathrm{a}$ & $462,2 \mathrm{~b}$ & $9,44 \mathrm{k}$ & $128 \mathrm{f}$ & $1,58 \mathrm{~d}$ & $2,63 c$ \\
\hline & BRS $257 \times$ BRS & $A$ & $\mathrm{MC}$ & 1 & & & & & & & \\
\hline \multirow[t]{2}{*}{ AL51 } & 258 & & & & 83,42 e & $11,00 \mathrm{~b}$ & $473,6 \mathrm{~b}$ & $9,68 k$ & $123 \mathrm{~h}$ & $2,93 \mathrm{c}$ & $2,58 \mathrm{c}$ \\
\hline & BRS $257 \times$ BRS & $A$ & $M$ & $\mathrm{D}$ & & & & & & & \\
\hline \multirow[t]{2}{*}{ AL52 } & 258 & & & & $72,75 \mathrm{f}$ & $10,75 \mathrm{~b}$ & $495,2 b$ & $11,75 \mathrm{j}$ & $125 \mathrm{~g}$ & $2,38 \mathrm{c}$ & $2,65 c$ \\
\hline & BRS $257 \times$ BRS & $A$ & $M$ & $\mathrm{D}$ & & & & & & & \\
\hline \multirow[t]{2}{*}{ AL53 } & 258 & & & & 83,33 e & $7,75 \mathrm{~b}$ & 720,8 a & $16,37 \mathrm{f}$ & $121 \mathrm{i}$ & $1,95 \mathrm{~d}$ & $2,90 \mathrm{~b}$ \\
\hline & BRS $257 \times$ BRS & $A$ & $M$ & & & & & & & & \\
\hline \multirow[t]{2}{*}{ AL54 } & 258 & & & 1 & $82,42 \mathrm{e}$ & $8,00 \mathrm{~b}$ & 797,5 a & $17,31 \mathrm{e}$ & $123 \mathrm{~h}$ & $2,30 \mathrm{c}$ & $2,98 \mathrm{~b}$ \\
\hline & BRS $257 \times$ BRS & $A$ & & & & & & & & & \\
\hline \multirow[t]{2}{*}{ AL55 } & 258 & & $A$ & $\mathrm{D}$ & 81,00 e & $9,50 \mathrm{~b}$ & $580,2 \mathrm{~b}$ & $17,57 \mathrm{e}$ & $123 \mathrm{~h}$ & $1,83 d$ & $2,90 \mathrm{~b}$ \\
\hline & BRS $257 \times$ & $A$ & M & & & & & & & & \\
\hline \multirow[t]{2}{*}{ AL56 } & MN01 & & & 1 & $93,58 d$ & $13,50 \mathrm{a}$ & 725,8 a & $17,86 \mathrm{e}$ & $121 \mathrm{i}$ & $2,03 d$ & $2,63 \mathrm{c}$ \\
\hline & BR $36 \times$ BRS & A & M & $\mathrm{D}$ & & & & & & & \\
\hline \multirow[t]{2}{*}{ AL57 } & 258 & & & & $92,00 \mathrm{~d}$ & $8,25 \mathrm{~b}$ & $731,7 \mathrm{a}$ & $16,34 \mathrm{f}$ & $123 \mathrm{~h}$ & $2,38 \mathrm{c}$ & $3,28 \mathrm{a}$ \\
\hline & BR $36 \times$ BRS & A & M & $\mathrm{D}$ & & & & & & & \\
\hline AL58 & 258 & & & & $85,25 d$ & $7,42 b$ & $821,7 \mathrm{a}$ & $18,21 \mathrm{e}$ & $123 \mathrm{~h}$ & $1,98 d$ & $3,18 \mathrm{a}$ \\
\hline AL59 & Late Giant TA & $A$ & $M$ & $\mathrm{D}$ & $76,50 \mathrm{f}$ & $7,67 \mathrm{~b}$ & $423,1 \mathrm{c}$ & $18,23 \mathrm{e}$ & $125 \mathrm{~g}$ & $2,70 \mathrm{c}$ & $2,28 d$ \\
\hline AL60 & ARA $\times$ BRS 155 & $A$ & $\mathrm{MC}$ & 1 & 84,42 e & $11,84 \mathrm{a}$ & 524,7 b & $9,81 \mathrm{k}$ & $128 \mathrm{f}$ & $2,08 d$ & $2,63 \mathrm{c}$ \\
\hline NK 7059 & & A & $M$ & 1 & $124,00 \mathrm{~b}$ & $14,83 \mathrm{a}$ & 910,9 a & $14,86 \mathrm{~h}$ & $120 \mathrm{j}$ & $2,28 d$ & $2,65 \mathrm{c}$ \\
\hline BMX & & $A$ & $\mathrm{MC}$ & 1 & & & & & & & \\
\hline Potência & & & & & 120,58 b & $12,84 \mathrm{a}$ & $806,7 \mathrm{a}$ & 17,53 e & $121 \mathrm{i}$ & $2,43 \mathrm{c}$ & $3,48 a$ \\
\hline BRS 257 & & $A$ & $\mathrm{MC}$ & $D$ & 83,83 e & $7,08 \mathrm{~b}$ & $796,1 \mathrm{a}$ & $16,84 \mathrm{f}$ & $120 \mathrm{j}$ & $1,75 \mathrm{~d}$ & $3,48 a$ \\
\hline BRS 258 & & A & $\mathrm{MC}$ & $\mathrm{D}$ & $70,42 \mathrm{f}$ & $9,33 \mathrm{~b}$ & $725,2 \mathrm{a}$ & $17,95 \mathrm{e}$ & $121 \mathrm{i}$ & $2,10 \mathrm{~d}$ & $3,10 \mathrm{~b}$ \\
\hline
\end{tabular}

${ }^{1}$ Médias seguidas pelas mesmas letras e na mesma coluna pertencem ao mesmo grupo pelo teste de Scott e Knott ao nível de $5 \%$ de probabilidade. $\mathrm{CT}$ : cor do tegumento; $\mathrm{CH}$ : cor do hilo; HC: hábito de crescimento; $\mathrm{A}$ : amarelo; $\mathrm{M}$ : marrom; $\mathrm{MC}$ : marrom claro; PI: preto imperfeito; D: determinado; I: indeterminado; SD: semi-determinado; ALT: altura de planta; APV: altura da primeira vagem; PRO: produção por parcela; PCS: peso de cem sementes; NDM: número de dias para maturação; ACA: acamamento e VAG: valor agronômico. 
As análises de variância revelaram diferenças significativas para todas as características quantitativas estudadas $(p<0,01)$, inferindo na existência de ampla variabilidade genética no conjunto de acessos (Tabela 2). Os coeficientes de variação ambiental $\left(\mathrm{CV}_{\mathrm{e}}\right)$ oscilaram entre 5,5 (PCS) e $27,7 \%$ (APV), sendo estes considerados baixos e semelhantes aos relatados por Silva et al. (2016) e Silveira et al. (2016), indicando alta qualidade experimental e, consequentemente, maior confiabilidade aos resultados obtidos.

Tabela 2. Resumo da análise de variância de sete características agronômicas avaliadas em 64 genótipos de soja.

\begin{tabular}{lllll}
\hline Característica $^{1}$ & QMT & p-valor & Média & $\mathrm{CV}_{\mathrm{e}}(\%)$ \\
\hline ALT & 1413,39 & $<0,01$ & 92,05 & 8,98 \\
APV & 36,86 & $<0,01$ & 11,43 & 27,71 \\
PRO & 81092,94 & $<0,01$ & 602,35 & 22,93 \\
PCS & 55,01 & $<0,01$ & 15,45 & 5,50 \\
NDM & 61,96 & $<0,01$ & 124,74 & 5,78 \\
ACA & 1,84 & $<0,01$ & 2,52 & 22,15 \\
VAG & 0,46 & $<0,01$ & 2,71 & 10,94
\end{tabular}

${ }^{1}$ ALT: altura de planta; APV: altura da primeira vagem; PRO: produção de grãos por parcela; PCS: peso de cem sementes; NDM: número de dias para maturação; ACA: acamamento; VAG: valor agronômico.

QMT: quadrado médio do tratamento; CV: coeficiente de variação ambiental

Para a característica ALT, o teste de Scott e Knott agrupou os acessos em seis diferentes grupos (Tabela 1). Grande parte dos genótipos (96,7\%) apresentaram ALT entre os valores das testemunhas comerciais estudadas $(70,42$ a 124,00 $\mathrm{cm})$, sendo que apenas os acessos AL14 (180,67 $\mathrm{cm})$ e AL16 $(169,83 \mathrm{~cm})$ apresentaram valores superiores. Segundo Sediyama (2009), plantas com altura entre 60 e $120 \mathrm{~cm}$ são as mais adequadas à colheita mecanizada. Todavia, plantas muito alta apresentam naturalmente tendência ao acamamento, o que pôde ser observado, uma vez que os acessos AL14 e AL16 apresentaram notas elevadas para essa característica. Por outro lado, a ALT não é considerada uma característica problemática quando a soja é colhida de forma manual como, por exemplo, quando as vagens são colhidas para edamame.

Em relação à APV, os acessos foram agrupados em apenas dois grupos pelo teste de Scott e Knott. Usualmente, busca-se obter cultivares com maior altura de inserção de primeira vagem (10 a $15 \mathrm{~cm}$ ), uma vez que são mais adequadas à colheita mecanizada (BEZERRA et al., 2017). Nesse sentido, as médias dos acessos foram alocadas nos mesmos grupos das testemunhas comerciais, que apresentaram médias entre 7,08 e $14,83 \mathrm{~cm}$. A característica ACA assume importante papel na seleção de genótipos, visto que junto com as características ALT e APV podem provocar perdas significativas na colheita mecanizada. $O$ teste de Scott e Knott alocou os acessos em quatro grupos, sendo que 29 acessos $(46,7 \%)$ se comportaram de forma tolerante e apenas seis $(9,4 \%)$ apresentaram forte tendência ao ACA.

Os acessos formaram doze grupos distintos para a característica PCS e os valores oscilaram entre 8,25 (AL16) e 26,83 g (AL08). O PCS é uma característica de grande importância na escolha do cultivar, uma vez que a aquisição de sementes de menor peso resultará em um menor custo de produção, além de apresentar maior velocidade nos processos de germinação e emergência (SOUZA, 2006). Por outro lado, grãos maiores são preferidos para os cultivares tipo alimento, principalmente para a fabricação de extrato hidrossolúvel de soja (leite de soja) e tofu, enquanto cultivares com grãos menores são recomendadas para a produção de natto (alimento fermentado) (SEDIYAMA, 2009).

Para PRO, as médias foram agrupadas em três grupos. Nenhum acesso superou estatisticamente as testemunhas comerciais, porém, 26 genótipos avaliados (43,3\%) apresentaram médias estatisticamente iguais às testemunhas. Para NDM, verificou-se que os 
acessos foram agrupados em dez grupos. Os genótipos apresentaram média entre 120 (NK 7059 e BRS 257) e 138 dias (AL34). Dentre os acessos avaliados, $25 \%$ destes foram alocados no mesmo grupo das cultivares comercias BMX Potência e BRS 258, que apresentaram médias de 121 dias. Genótipos com menor NDM são desejados em programas de melhoramento de soja, uma vez que cultivares precoces podem ser indicadas para áreas de renovação de canaviais (BIZARI, 2014), além de serem recomendadas em regiões onde a segunda safra é tradicional.

Pela análise de importância de caracteres de Singh (1981) é possível classificar as variáveis estudadas de acordo com suas respectivas contribuições para a divergência genética total e, assim, identificar as características que mais e menos contribuem para discriminação dos genótipos. Pode-se observar que a característica PRO $(16,7 \%)$ foi a mais eficiente na discriminação dos acessos avaliados, enquanto que a característica ALT (8,9\%) apresentou menor contribuição (Tabela 3). Este resultado é de fundamental importância em estudos de divergência genética, pois no processo de escolha dos genitores, além da dissimilaridade entre os genótipos, também deve-se considerar $o$ alto rendimento de grãos para a obtenção de progênies superiores. Resultados divergentes foram relatados por Almeida et al. (2011) e Glasenapp et al. (2014), em que a PRO apresentou pouca influência na discriminação de genótipos de soja tipo grão em estudos de diversidade.

Tabela 3. Contribuição relativa de sete características para dissimilaridade genética de 64 genótipos de soja por meio método proposto por Singh (1981).

\begin{tabular}{lc}
\hline Característica & Valor (\%) \\
\hline Produção de grãos por parcela & 16,71 \\
Acamamento & 16,59 \\
Altura da primeira vagem & 15,39 \\
Valor agronômico & 15,34 \\
Número de dias para maturação & 14,74 \\
Peso de cem sementes & 12,27 \\
Altura de planta & 8,96 \\
\hline
\end{tabular}

O agrupamento hierárquico UPGMA obteve maior valor de correlação cofenética $(0,81)$ quando comparado com os métodos de agrupamento de Ward $(0,64)$ e Vizinho Mais Próximo $(0,71)$. De acordo com Sokal e Rohlf (1962), valores de correlação cofenética superiores a 0,8 são desejados, pois infere em um bom ajuste entre as matrizes de distância e de agrupamento. Além disso, a correlação cofenética entre essas matrizes, utilizando o método UPGMA, foi altamente significativa pelos testes $t$ e Mantel, recomendando-se, assim, o agrupamento UPGMA para a discussão dos resultados.

A realização de um corte na distância 0,2 do dendrograma, conforme resultado obtido pelo método de Mojena (1977), possibilitou a formação de seis grupos distintos (Figura 1). O grupo I foi formado pelos genótipos AL14 e AL16. Estes dois acessos são originados do mesmo cruzamento (21 $\times 9$ RC2F10) e são caracterizados por apresentarem média elevada de AP e baixa de APV. O grupo II é constituído pelos acessos AL12 e AL13, ambos provenientes do mesmo cruzamento (Wilami $\times$ AP). Dentre todos os acessos avaliados, estes dois genótipos foram os únicos a apresentarem coloração de tegumento marrom, além de possuírem valores elevados de PRO. O grupo III é composto apenas pelo acesso AL23, provavelmente por ser o único genótipo originado do cruzamento entre Toffu I e ARA. Esse grupo é caracterizado por apresentar médias elevadas de AP, APV e PRO, além de ser o único acesso de hábito de crescimento semi-determinado. 
Figura 1. Dendrograma da dissimilaridade genética entre 64 acessos de soja obtido pelo método UPGMA com base no algoritmo de Gower. O ponto de corte na distância 0,20 foi obtido por meio do método de Mojena (1977) gerando, assim, a formação de seis diferentes grupos.

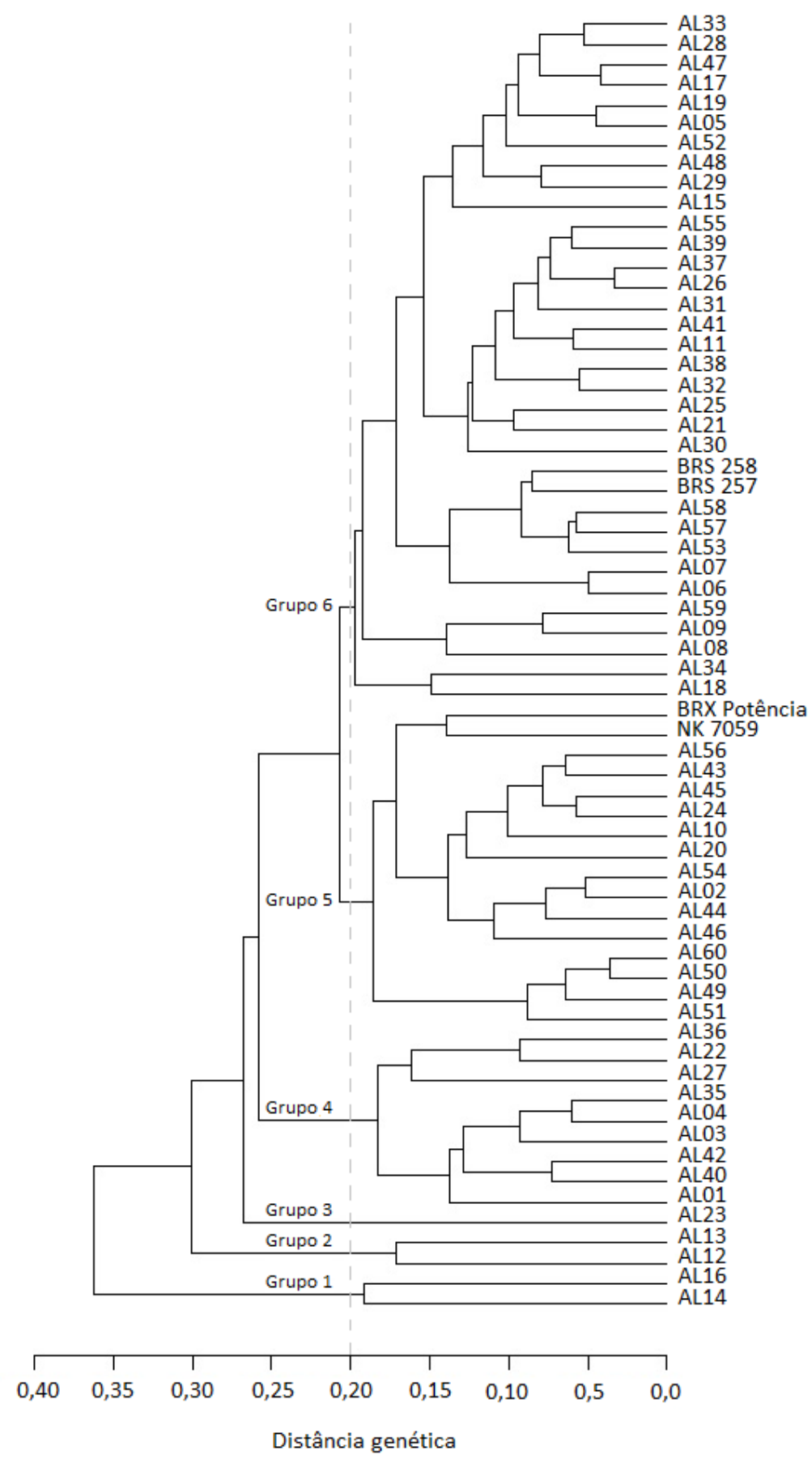

No grupo IV, formado por nove acessos, os mesmos são caracterizados por apresentarem hábito de crescimento determinado e com médias elevadas das características APV e ACA, além de apresentarem médias baixas para PRO e VAG. Por apresentar tais características, o grupo IV pode ser considerado $o$ de menor potencial para cruzamentos futuros. Os grupos V e VI foram constituídos por 16 e 34 acessos, respectivamente. As cultivares NK 7059 e BMX Potência foram alocados no grupo $V$, enquanto que as cultivares BRS 257 e BRS 258 foram agrupadas no grupo VI. De modo geral, os dois grupos comportarem-se de forma semelhante para as características 
quantitativas. Todavia, a característica hábito de crescimento foi determinante na discriminação dos dois grupos, uma vez que os acessos do grupo $V$ são caracterizados por apresentarem hábito de crescimento indeterminado, enquanto os acessos do grupo VI do tipo determinado.

A formação de grupos é de fundamental importância para a escolha dos genitores, pois as novas combinações híbridas devem ser baseadas na magnitude de suas dissimilaridades e no potencial per se dos genitores. Neste sentido, as combinações AL06 × AL22 e AL07 × AL22 são promissoras para desenvolvimento de genótipos superiores destinados ao consumo in natura (saladas) e na produção de leite de soja e tofu, uma vez que apresentam CT e CH claro, PCS elevados e características agronômicas favoráveis. A combinação AL13 $\times$ AL60, por aliar baixo PCS e elevada PRO, mostra-se promissora para produção de natto. Em relação ao desenvolvimento de genótipos destinados à produção de kuromame, a utilização dos acessos AL12 e AL13 como genitores mostra-se vantajosa pela presença de $\mathrm{CT}$ e $\mathrm{CH}$ marrons, uma vez que essa cor é favorável para tal destino, possibilitando, assim, ampliar o número de recombinantes desejáveis.

\section{CONCLUSÕES}

As características quantitativas e qualificadas avaliadas indicaram ampla variabilidade genética entre os acessos avaliados. $\mathrm{A}$ característica produtividade foi a mais eficiente na discriminação dos acessos avaliados, enquanto que a característica altura de planta apresentou menor contribuição. O método de Gower foi eficiente na discriminação dos grupos, demonstrando que a análise simultânea de dados qualitativos e quantitativos é viável e pode permitir maior eficiência no conhecimento da divergência entre acessos de bancos de germoplasma. As hibridações AL06 $\times$ AL22 e AL07 × AL22 são promissoras para obtenção de genótipos superiores destinados ao consumo in natura e produção de leite de soja e tofu. A combinação AL13 $\times$ AL60 é considerada favorável para obtenção de genótipos destinados à produção de natto. A utilização dos acessos AL12 e AL13 como genitores é vantajosa para obtenção de genótipos destinados à produção de kuroname.

\section{REFERÊNCIAS}

ALMEIDA, R.A.; PELUZIO, J.M.; AFFÉRRI, F.S. Divergência genética entre cultivares de soja, sob condições de várzea irrigada, no sul do Estado Tocantins. Revista Ciência Agronômica, v. 42, n. 1, p. 108-115, 2011. https://doi.org/10.1590/S1806$\underline{66902011000100014}$

BEZERRA, A.R.G.; SEDIYAMA, T.; DA SILVA, F.L.; BORÉM, A.; SILVA, A.F.; SILVA, F.C.S. Agronomical Aspects of the Development of Cultivars. In: SILVA, F. L. et al. (Ed.) Soybean breeding. Springer, 2017, 440p. https://doi.org/10.1007/978-3-319-57433221

BIZARI, E.H. Índice de seleção para caracteres agronômicos em populações segregantes de soja. 2014, 32f. Dissertação (Mestrado em Genética e Melhoramento de Planta) - Universidade Estadual de São Paulo, 2014.

CARRÃO-PANIZZI, M.C.; BERTGNOLLI, P.F.; MOREIRA, J.U.V.; DA COSTA, L.C.; CARAFFA, M.; COSTAMILAN, L.M.; STRIEDER, M.L. Melhoramento de soja para alimentação humana na Embrapa Trigo - safra agrícola. Soja: resultados de pesquisa 2015/2016. 2016, 25 p.

CRUZ, C.D. Genes Software-extended and integrated with the R, Matlab and Selegen. Acta Scientiarum. Agronomy, v.38, n.44, p.547-552, 2016.

http://dx.doi.org/10.4025/actasciagron.v38i4.3262 9

FEHR, W.P.; CAVINESS, C.E. Stages of soybean development. Agriculture and Home Economics Experiment Station and Cooperative Extension Service. Ames: lowa State University, 1977. 11 p. (Special Report; 80).

GLASENAPP, J.S.; SEDIYAMA, T.; CRUZ, C.D.; MATSUO, E.; BROMMONSCHENKEL, S.H.; OLIVEIRA, R.D.C.T.; HAMAWAKI, O. Diversidade de características agronômicas e moleculares em cultivares de soja com diferentes graus de resistência à Phakopsora pachyrhizi. Bioscience Journal, v.31, n.1, p.25-36, 2014. https://doi.org/10.14393/BJ-v31n1a2015-171666 
GONÇALVES, L.S.A.; RODRIGUES, R.; AMARAL JÚNIOR, A.T.; KARASAWA, M.; SUDRÉ, C.P. Comparison of multivariate statistical algorithms to cluster tomato heirloom accessions. Genetics and Molecular Research, v.7, n.1, p.1289-1297, 2008. https://doi.org/10.4238/vol7-4gmr526

GOWER, J.C.A. A general coefficient of similarity and some of its properties. Biometrics, v.27, n.4, p.857-874, 1971. https://doi.org/10.2307/2528823

HEA, F.J.; CHEN, J.Q. Consumption of soya bean: the link to decreased breast cancer incidence. Oilseeds Focus, v. 3, n. 1, p. 34-37, 2017.

HIRAKURI, M.H.; LAZZAROTTO, J.J. O agronegócio da soja nos contextos mundial e brasileiro. Londrina: Embrapa Soja, 2014. 70 p. (Documentos; 349).

KYRIAKOPOULOU, O.G.; ARENS, P.; PELGROM, K.T.; KARAPANOS, I.; BEBELI, P.; PASSAM, H.C. Genetic and morphological diversity of okra (Abelmoschus esculentus [L.] Moench.) genotypes and their possible relationships, with particular reference to Greek landraces. Scientia Horticulturae, v. 171, n.1, p.58-70, 2014. https://doi.org/10.1016/j.scienta.2014.03.029

MANTEL, N. The detection of disease clustering and a generalized regression approach. Cancer research, v. 27, n. 1, p. 209-220, 1967.

MILLIGAN, G.W.; COOPER, M.C. An examination of procedures for determining the number of clusters in a data set. Psychometrika, v. 50, n. 1, p. 159179, 1985. https://doi.org/10.1007/BF02294245

MOJENA, R. Hierarchical grouping methods and stopping rules: an evaluation. The Computer Journal, v.20, n.1, p.359-363, 1977. https://doi.org/10.1093/comjnl/20.4.359

MOURA, M.D.C.C.; GONÇALVES, L.S.A.; SUDRÉ, C.P.; RODRIGUES, R.; DO AMARAL JÚNIOR; A.T.; PEREIRA, T.N. The Grower's algorithm on the estimate of genetic diversity in chili pepper germoplasm. Horticultura Brasileira, v. 28, n. 2, p. 155-161, 2010. http://dx.doi.org/10.1590/S0102$\underline{05362010000200003}$
OLIVEIRA, R.L.; GONÇALVES, L.S.A; RODRIGUES, R.; BABA, V.Y.; SUDRÉ, C.P.; DOS SANTOS, M.H.; ARANHA, F.M. Genetic divergence among pumpkin landraces. Semina: Ciências Agrárias, v. 37, n.2, p. 547-556, 2016. http://dx.doi.org/10.5433/1679$\underline{0359.2016 v 37 n 2 p 547}$

ROCHA, M.C.; GONÇALVES, L.S.A.; RODRIGUES, R.; SILVA, P.R.A.D.; CARMO, M.G.F.D; ABBOUD, A.C.D.S. Uso do algoritmo de Gower na determinação da divergência genética entre acessos de tomateiro do grupo cereja. Acta Scientiarum. Agronomy, v. 32, n. 3, p. 423-431, 2010.

http://dx.doi.org/10.4025/actasciagron.v32i3.4888

SCOTT, A.J.; KNOTT, M.A. Cluster analysis methods for grouping means in the analysis of variance. Biometrics, v.30, n.3, p.507-512, 1974. https://doi.org/10.2307/2529204

SEDIYAMA, T. Tecnologia de produção da soja. Londrina: Mecenas, 2009. 314 p.

SILVA, F.C.S.; SEDIYAMA, T.; DA SILVA, A.F.; BEZERRA, A.E.R.G.; ROSA, A.D.P.; FERREIRA, L.V.; $C R U Z, C . D$. Identification of new descriptors for differentiation of soybean genotypes by Gower algorithm. African Journal of Agricultural Research, v.11, n.11, p.961-966, 2016. https://doi.org/10.5897/AJAR2015.10158

SILVEIRA, D.A.; PRICINOTTO, L.F.; NARDINO, M.; BAHRY, C.A.; PRETE, C.E.C.; CRUZ, L. Determination of the adaptability and stability of soybean cultivars in different locations and at different sowing times in Paraná state using the AMMI and Eberhart and Russel methods. Semina: Ciências Agrárias, v.37, n.6, p.3973-3982, 2016. https://doi.org/10.5433/16790359.2016v37n6p3973

SINGH, D. The relative importance of characters affecting genetic divergence. The Indian Journal of Genetic and Plant Breeding, v. 41, n. 2, p. 237-245, 1981.

SOKAL, R.R.; ROHLF, F.J. The comparison of dendrograms by objective methods. Taxon, v. 11, 
n.2,

p.33-40,

1962.

https://doi.org/10.2307/1217208

SOUZA, E.L. Qualidade de sementes de soja comercializadas pela cooperativa agroindustrial COPAGRIL no Paraná. 2006, 34f. Dissertação (Mestrado em Ciência e Tecnologia de Sementes) Universidade Federal de Pelotas, Pelotas, 2006.

YOKOMIZO, G.K.I.; DUARTE, J.B.; VELLO, N.A. Correlações fenotípicas entre tamanho de grãos e outros caracteres em topo-cruzamentos de soja tipo alimento com tipo grão. Pesquisa Agropecuária Brasileira, v. 35, n. 11, p. 2235-2241, $2000 . \quad \quad$ https://doi.org/10.1590/\$0100-

$\underline{204 \times 2000001100016}$

Recebido para publicação em 23/11/2018

Revisado em 11/01/2019

Aceito em 11/01/2019 